\title{
Latitudinal Variation of Aerosol Sizes Inferred from Titan's Shadow ${ }^{1}$
}

\author{
ERICH KarkoschKa AND RALPh D. LORENZ \\ Lunar and Planetary Laboratory, University of Arizona, Tucson, Arizona 85721-0092 \\ E-mail: erich@pirl.lpl.arizona.edu
}

Received April 24, 1996; revised August 30, 1996

\begin{abstract}
We measured the location, size, and shape of Titan's shadow in five images taken with the Hubble Space Telescope in 1995. We inferred the altitude of Titan's optical limb at wavelengths of 337-954 $\mathrm{nm}$ to an accuracy of $15 \mathrm{~km}$. At each wavelength, altitudes are constant north of $-5^{\circ}$ and also constant but lower south of $-50^{\circ}$ latitude, with a linear transition region inbetween. The amplitude of the variation of altitude with latitude increases from close to zero at wavelength $337 \mathrm{~nm}$ to $130 \mathrm{~km}$ at $954 \mathrm{~nm}$. We conclude that Titan's aerosols are larger $(0.3 \mu \mathrm{m}$ mean radius) at northern latitudes where we probe the detached haze layer than at southern latitudes $\left(0.1 \mu \mathrm{m}\right.$ south of $\left.-50^{\circ}\right)$ where we probe the main haze layer below. The geometric cross section of aerosols at probed altitudes $(\sim 300 \mathrm{~km})$ does not show a significant latitudinal variation. The wavelength dependence of the size of Titan's shadow is not consistent with a spherical shape of aerosols, but gives evidence of their fractal nature. $\odot 1997$ Academic Press
\end{abstract}

\section{INTRODUCTION}

Titan's aerosol sizes have been inferred by different techniques. Tomasko and Smith (1982) used Pioneer 11 polarimetry to derive a mean aerosol radius of $0.1 \mu \mathrm{m}$. Rages et al. (1983) found a lower bound of $0.2 \mu \mathrm{m}$ based on Voyager images at high phase angles. Both determinations assumed spherical aerosols, and the disagreement is an indication that Titan's aerosols are not spherical.

The variation of altitude at a certain optical depth with wavelength yields information about the wavelength dependence of optical depth, and this constrains aerosol sizes. Smith (1980) measured Titan's radius at 440 and $640 \mathrm{~nm}$ wavelength using Pioneer 11 data. His data give a decrease of altitude of the optical limb by $40 \pm 33 \mathrm{~km}$ from the blue to the red wavelength. A decreasing altitude with increasing wavelength indicates aerosols smaller than 0.3 $\mu \mathrm{m}$ radius. Smith et al. (1981) reported the altitude of

\footnotetext{
${ }^{1}$ Based on observations with the NASA/ESA Hubble Space Telescope obtained at the Space Telescope Science Institute, which is operated by the Association of Universities for Research in Astronomy, Incorporated, under NASA contract NAS5-26555.
}

Titan's optical limb from Voyager 1 images, although no wavelength dependence was investigated. Using a high phase-angle image in the clear filter $(480 \mathrm{~nm})$ from Voyager 2, Rages and Pollack (1983) found the altitude of the optical limb higher at the equator than at the poles.

Imaging of Titan by the Hubble Space Telescope has the advantage of a wide wavelength range. However, limb profiles of these images are greatly affected by the point spread function of the telescope and by the limb darkening profile, both of which make measurements of the altitude of the optical limb difficult.

Hubbard et al. (1993) constrained Titan's haze properties by a stellar occultation in 1989. They determined an aerosol size of $0.2 \mu \mathrm{m}$, or a smaller mean size if the variance of the size distribution is large. They found a north-south asymmetry of the altitude of the optical limb with the transition at $-20^{\circ}$ latitude. Since occultation data are very sensitive to the gas density and density gradient, haze properties were constrained after gas density and density gradient had been modeled and accounted for.

Here, we report on a unique way to constrain the altitudes and sizes of Titan's aerosols. We use the location, size, and shape of Titan's shadow cast on Saturn in images over a wide wavelength range (337-954 nm). Our images taken in 1995 are the only well-resolved images of Titan's shadow so far. Hubbard et al. (1996) report imaging Titan's shadow on Mimas and Saturn's rings, but seeing from the 1.8-m Vatican Advanced Technology Telescope was 1.3 arc-seconds. The next opportunity to image Titan's shadow will not come before 2008 .

Previous methods of determining the size of Titan's aerosols required sophisticated modeling of Titan's atmosphere with the need to assume the values for poorly known parameters about Titan's atmosphere. Only recently has there been any attempt to model a latitudinal variation of aerosol sizes (Hutzell et al. 1996). On the other hand, by looking at the shape of Titan's shadow at different wavelengths, one can almost "see" that aerosol sizes must considerably vary with latitude without knowledge of any parameter of Titan's atmosphere.

We now describe our observations. Following are sec- 
TABLE I

Observational Data

\begin{tabular}{|c|c|c|c|c|c|c|c|c|c|c|c|c|}
\hline Filter & $\begin{array}{c}\text { Wavl. } \\
\text { (nm) }\end{array}$ & $\begin{array}{c}\mathrm{UT} \\
(\mathrm{h}: \mathrm{m}: \mathrm{s})\end{array}$ & $\begin{array}{l}\text { Exp. } \\
(\mathrm{sec})\end{array}$ & $\begin{array}{c}\text { Long. } \\
\left({ }^{\circ}\right)\end{array}$ & $\begin{array}{c}\text { Lat. } \\
\left(^{\circ}\right)\end{array}$ & $\begin{array}{l}H_{\mathrm{N}} \\
(\mathrm{km})\end{array}$ & $\begin{array}{l}H_{\mathrm{S}} \\
(\mathrm{km})\end{array}$ & $\begin{array}{c}\sigma \\
(\mathrm{km})\end{array}$ & $n_{\mathrm{r}}$ & $n_{i}$ & $Q_{.3}$ & $Q_{.1}$ \\
\hline F336W & 337 & $13: 11: 52$ & 70 & 38.1 & -36.49 & 344 & 334 & \pm 6 & 1.635 & 0.18 & 2.71 & 2.11 \\
\hline F467M & 467 & $13: 15: 24$ & 16 & 36.5 & -36.47 & 318 & 282 & \pm 4 & 1.715 & 0.078 & 4.00 & 1.52 \\
\hline FQCH4N-B & 620 & $13: 19: 31$ & 30 & 34.7 & -36.45 & 311 & 249 & \pm 5 & 1.690 & 0.0225 & 3.14 & 0.74 \\
\hline F673N & 673 & $13: 22: 26$ & 18 & 33.5 & -36.43 & 312 & 242 & \pm 5 & 1.680 & 0.0150 & 3.09 & 0.57 \\
\hline F953N & 954 & $13: 26: 06$ & 100 & 31.9 & -36.42 & 306 & 178 & \pm 10 & 1.655 & 0.0024 & 2.35 & 0.10 \\
\hline
\end{tabular}

Note. Wavl. is the mean wavelength. UT is the Universal Time on August 6, 1996 at mid-exposure. Exp. is the exposure time. Long. and Lat. are assumed coordinates of the shadow of Titan's center. Longitudes are measured from the central meridian. $H_{\mathrm{N}}$ and $H_{\mathrm{S}}$ are observed altitudes of the optical limb for the northern and southern regions. $\sigma$ is the standard deviation of data points from the fit (systematic errors are estimated at $15 \mathrm{~km}) . n_{\mathrm{r}}$ and $n_{\mathrm{i}}$ are assumed real and imaginary refractive indices of Titan's aerosols. $Q_{.3}$ and $Q_{.1}$ are extinction efficiencies for aerosols of mean radii 0.3 and $0.1 \mu \mathrm{m}$.

tions of our modeling and a discussion. A summary concludes this work.

\section{OBSERVATIONS}

On August 6, 1995, the Planetary Camera of the Hubble Space Telescope took five images of Saturn containing Titan's shadow for an observing program by Martin Tomasko and Erich Karkoschka (program ID 6030). Table I lists the filter, mean wavelength, observing time, exposure time, and shadow location for each image. The mean wavelength was calculated by the solar flux spectrum of Neckel and Labs (1984), by Saturn's albedo spectrum (Karkoschka 1994), and by filter and system response functions (Burrows et al. 1995). Saturn's phase angle was $3.93^{\circ}$. The Sun was $1.56^{\circ}$ above Saturn's ring plane, the Earth $0.12^{\circ}$ below the plane. The image scale was $291 \mathrm{~km} /$ pixel. Figure 1 displays the image at $620 \mathrm{~nm}$ wavelength.

The F336W filter has a red leak near $700 \mathrm{~nm}$ which amounts to $3.5 \%$ for light reflected from Saturn. The contribution of the leak was estimated from the two images of longest wavelengths and subtracted.

Flatfielded images were deconvolved by the Wiener method using point spread functions calculated by the Tiny Tim software of the Space Telescope Science Institute (Krist 1994). The Wiener method uses the Fourier transform technique and allows the user to specify the point spread function of the deconvolved image. We specified a $3 \times 3$ pixel point spread function, roughly resembling the core of the point spread function of the raw image. This caused a very moderate sharpening of the images and increased the noise only slightly.

The deconvolved images theoretically concentrate $100 \%$ of the total light of a point source into a $3 \times 3$ pixel area. However, nonzero counts off the disk of Saturn indicate that a small fraction of light is scattered smoothly over an area of several arc-seconds in the deconvolved images. A similar, small amount of background level is seen in the center of Titan's shadow. Figure 2 displays east-west scans across Titan's shadow.

In order to measure the radius of the shadow as a function of position angle, one needs to know or assume the location of the shadow of Titan's center of mass (where the line connecting the centers of the Sun and Titan intercepts Saturn's optical surface). We assumed an east-west location which yields the same altitudes for Titan's morning and evening limbs. We do not expect a variation between both limbs since radiative time scales in the region of Titan's atmosphere we probe are much longer than a Titan day (Flasar et al. 1981).

We measured north-south coordinates relative to the very sharp, nearly edge-on ring. We took into account that the scale and distortion of the camera changes slightly as a function of wavelength according to coefficients given by Trauger et al. (1995).

To the east and west of Titan's shadow, Saturn displayed a banded structure. Longitudinal features are barely visible (cf. Figs. 1 and 2). They have contrasts of $\sim 1 \%$ or less. For each filter and at each latitude, we fitted intensities measured east and west of the shadow by a Linear Limb Darkening function (cf. Karkoschka and Tomasko 1993). The data numbers of that latitude were then divided by the fitted function. This yielded values close to unity outside the shadow and almost constant values near zero in the umbra. In-between, there is a transition region of $\sim 3$ pixel width, the penumbra. The penumbra is small compared to the shadow diameter of about 20 pixels. Nevertheless, its finite size must be considered. Systematic effects such as this one are discussed in Section III. Now, we estimate random errors of our measurements.

\section{Precision}

By bilinear and by bicubic interpolation between pixel centers, we determined the curve where the value of the 


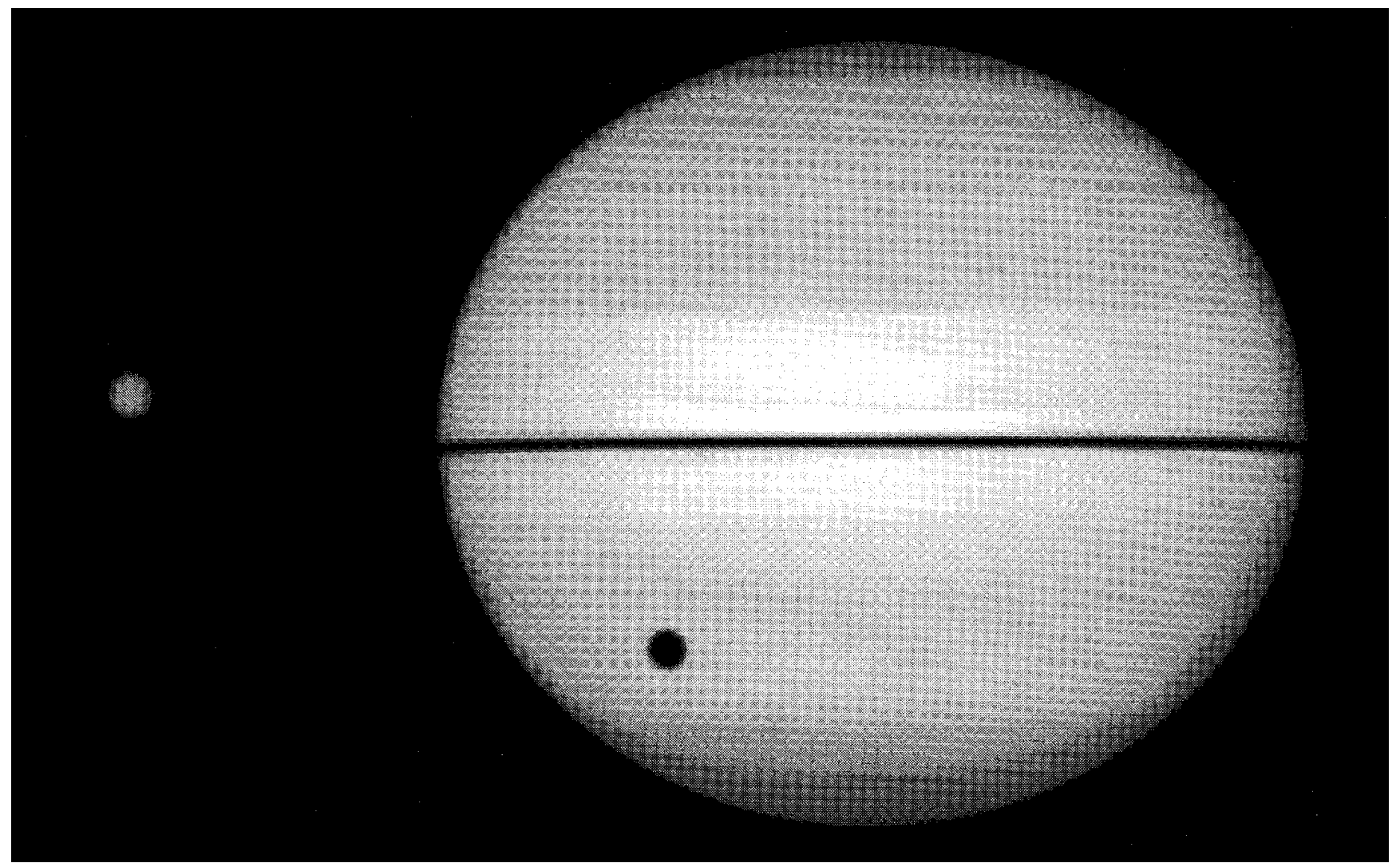

FIG. 1. Titan casting its shadow onto Saturn. Saturn's rings were almost edge-on. The dark lane below Saturn's equator is the shadow of the rings. This image was taken with a filter centered at wavelength 620-nm.

divided data numbers reach halfway between unity and the constant near zero. Both interpolations yielded similar curves. The standard deviation between both curves is 0.02 pixel. Since the width of the penumbra is only three pixels wide, linear interpolation produces radial intensity profiles

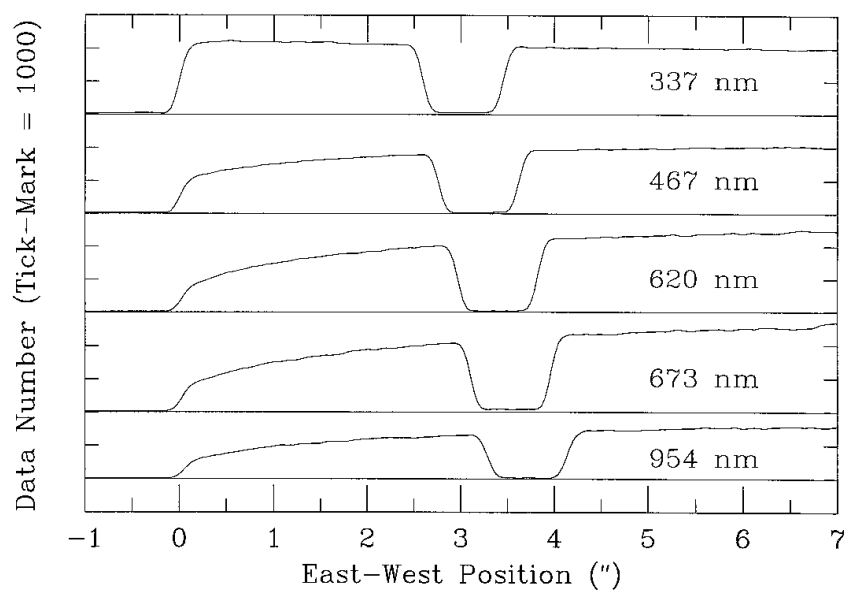

FIG. 2. Scans along Saturn latitude $-36.5^{\circ}$ across the center of Titan's shadow. Mean wavelengths of the five filters are indicated. with kinks depending on the fractional pixel position of the $50 \%$ point. On the other hand, radial intensity profiles calculated with cubic interpolation yields smooth curves which are almost identical, almost independent of the sampling. The deviation between both interpolation methods of 0.02 pixel is mostly due to the lower accuracy of linear interpolation. Therefore, we base our results on the more accurate bicubic interpolation.

Pixels near $50 \%$ intensity have data numbers near 1000 or 15,000 detected photons, corresponding to a signal/noise level of 120 (original image) or 80 (deconvolved image). Considering the intensity gradient, this corresponds to a random error of about $8 \mathrm{~km}$ in the measured radius.

The total contribution of all random errors can be estimated from the data. We compared all data points between the morning and evening terminators at the same latitude. We also compared the data points with a simple latitudinal function described in Section III. Both comparisons give a standard deviation of single measurements of about 8 $\mathrm{km}$ (cf. " $\sigma$ " in Table I). In principle, this is only an upper limit on the random error, since some of the apparent noise could be real. The determination of the random error of $8 \mathrm{~km}(0.03$ pixel $)$ is consistent with the estimate above derived from the signal/noise of the data numbers. 
In the past, we have used the same bicubic interpolation to measure the position of features in other exposures with the same PC1 camera. For example, we measured the radius of several sections of the eccentric $\varepsilon$-ring of Uranus. We found a standard deviation of 0.04 pixel between our measurements and the predicted locations based on stellar occultation data. Since the predicted positions are very accurate (corresponding to 0.01 pixel), the deviation of 0.04 pixel gives our total (random plus systematic) errors. This number is slightly larger than our random error for measurements of Titan's shadow. This is expected since the Uranian ring was exposed to low data numbers only. Note that all our measurements were restricted to the central area of the PC1 chip. It may be more difficult to achieve a similar accuracy over a larger area of the chip.

Each shadow has a circumference of about 60 pixels. Averaging one hemisphere reduces the random error to 2 $\mathrm{km}$. However, as we will see in the following section, the accuracy of our results is limited by our ability to account for systematic errors and not by random errors.

\section{MODELING THE SHAPE OF THE SHADOW}

\section{Geometric Magnification}

If the Sun were a point source and Titan a sphere without atmosphere, Titan's shadow on Saturn would have been 1.00085 times its physical size. This number simply derives from geometric optics taking into account the distance between Titan and Saturn's atmosphere $(1,215,000 \mathrm{~km})$ and the distance to the Sun (8.825 AU). This effect increases the radius of the shadow by $2.5 \mathrm{~km}$.

\section{Size of the Sun}

The finite size of the Sun shifts the location of $50 \%$ intensity inward. An observer on Saturn watching the center of the solar disk just occulted by Titan sees more than half of the solar disk. For the observer, the Sun appears about five times smaller than Titan. The solar radius projected into Titan's distance is $588 \mathrm{~km}$, compared to 2800 $2900 \mathrm{~km}$ of Titan's radius including the visible haze. A simple geometric calculation shows that the observer must move $20 \mathrm{~km}$ inward in order to see half of the solar disk.

The visibility of half of the solar disk is not identical with the visibility of half of the solar radiation because of the limb darkening of the Sun. More than half of the brighter, center area is covered, but less than half of the limb. In order to receive half of the solar radiation, the observer must move slightly, depending on the limb darkening at each wavelength. Limb darkening data by Allen (1973) were interpolated for the wavelength of each filter. Integrations over the unocculted part of the solar disk yielded the shift of the $50 \%$ intensity location due to the finite size of the Sun. At wavelength $337 \mathrm{~nm}$, where solar limb darkening is strong, the shift is $16 \mathrm{~km}$; wavelength at $954 \mathrm{~nm}$, where limb darkening is less significant, the shift is $19 \mathrm{~km}$.

\section{Refraction}

Refraction in Titan's atmosphere at altitude $200 \mathrm{~km}$ is $\sim 2^{\prime \prime}$ and thus much smaller than the size of the solar disk seen from Saturn $\left(200^{\prime \prime}\right)$. Therefore, refraction has no measurable effect on the size of the shadow.

\section{Scattering in Saturn's Hazes}

Solar photons which reach Saturn's atmosphere are scattered once or multiple times at different altitudes in Saturn's atmosphere before they go on their way to Earth. They leave Saturn's atmosphere at a different location than where they entered, causing the edge of the shadow to smear even if it were sharp otherwise. We estimate the dislocation is on the order of two scale heights of Saturn's atmosphere, or about $40 \mathrm{~km}$. This "smearing" effect is much smaller than the size of the penumbra $(\sim 800 \mathrm{~km})$, and our modeling confirms that it changes the $50 \%$ location by much less than $1 \mathrm{~km}$. Thus it was neglected.

\section{Geometric Distortion}

The finite phase angle of $4^{\circ}$ changes the apparent size and shape of the shadow. A spherical Titan casts a shadow which is close to cylindrical in the vicinity of Saturn. The intersection of this cylinder with the optical surface of Saturn yields a highly elongated shadow on Saturn (axis ratio $\sim 1.5$ ). We calculated the location for several radii of Titan and in intervals of $1^{\circ}$ in position angle, using Saturn's ellipsoidal shape by Davies et al. (1992). When viewed from Earth, this curve appears close to circular (apparent axis ratio 1.1 ) because the foreshortening seen from Earth and Sun are similar. We performed this calculation for each image separately, since the distortion varied over the time interval of the observations as Titan's shadow moved across Saturn's disk (cf. Fig. 2).

\section{Smear due to Motion}

The Hubble Space Telescope was tracking Saturn, while Titan's shadow moved. This caused a smear in the eastwest direction. For the longest exposure (100 sec), the corresponding smear causes the $50 \%$ point to move $4 \mathrm{~km}$ inward in the north-south direction according to synthetic intensity profiles. For the 70 -sec exposure this effect is half as large. It is negligible for the other exposures. We corrected our measurements for the smear.

We also have a long, 500-sec exposure of Titan's shadow at wavelength $890 \mathrm{~nm}$. Our measurements with synthetic intensity profiles smeared to that extent give a systematic effect of $90 \mathrm{~km}$ in the north-south direction. Indeed, the 
measurements on the real shadow give a north-south radius about $90 \mathrm{~km}$ smaller than for our $954-\mathrm{nm}$ image. Thus, we have a check on the validity of our applied corrections. Because of the large systematic effect for an image with 500 -sec exposure time, we excluded it from this work.

\section{Point Spread Function}

The point spread function of the deconvolved images is 3 pixels wide. Synthetic profiles show that this causes the $50 \%$ point to move $13 \mathrm{~km}$ inward. We corrected our data accordingly.

\section{Rayleigh Scattering}

According to models by Toon et al. (1992), the Rayleigh extinction is two orders of magnitude below that of Titan's aerosols for the considered altitudes and wavelengths. Thus, we neglected Rayleigh extinction in further considerations. Even if the ratio between both extinctions is only one instead of two orders of magnitudes, it does not change any of our results.

\section{Diffraction}

The size of the diffraction pattern of Titan's shadow is on the order of the square root of the product of wavelength and Titan-Saturn distance. This value is less than $0.05 \mathrm{~km}$ and completely negligible.

\section{CCD Defect}

Every 34th row of the CCDs of the Wide Field/Planetary Camera 2 is $3 \%$ too narrow (Biretta 1995). Row 304 of the PC1 chip is such a row. The maximum positional error occurs at the adjacent rows, and it is 0.015 pixel, or $4 \mathrm{~km}$. We corrected for this small, systematic positional shift.

\section{Centering}

While Titan's absolute location cannot be predicted to an accuracy of a few kilometers, its relative positions are accurately known within our 25-min. observing interval from its orbital velocity vector. Taking into account the changing angle of the Sun above Saturn's equatorial plane during our observing time, Titan's shadow moved north by $0.07^{\circ}$ in latitude between our first and last exposure (cf. Table I).

Our measurements indicate that Titan's optical limb is almost circular at wavelength $337 \mathrm{~nm}$, but increasingly elongated at longer wavelengths with the equatorial diameter larger than the polar diameter. Therefore, we assumed that Titan's center of gravity is almost centered within the optical limb at wavelength $337 \mathrm{~nm}$, but not necessarily at other wavelengths. We off-centered the 337nm wavelength shadow by about 10 times less than the 954-nm wavelength shadow, since we observe the same

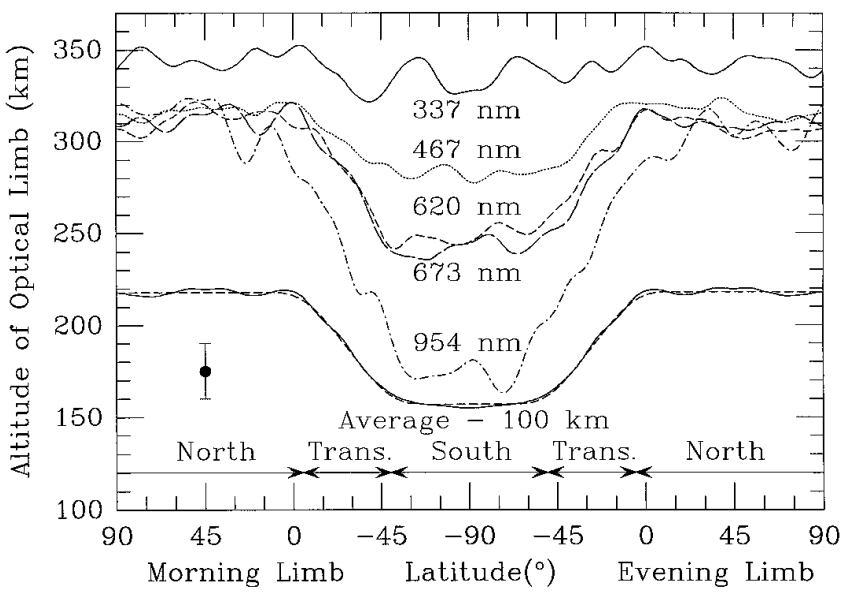

FIG. 3. Altitude of the optical limb as function of position along the terminator for the five filters of this study. The shape of the latitudinal variation of altitude is best seen in the average of the five upper curves, displaced by $100 \mathrm{~km}$ (solid curve at bottom). For this curve, morning and evening limbs were averaged too. The altitude is essentially constant northward of $-5^{\circ}$ (northern region) and southward of $-50^{\circ}$ latitude (southern region), with a linear transition region inbetween, as shown by the dashed fit. The size of the systematic error of $15 \mathrm{~km}$ is shown at lower left. Random variations are obvious in each curve.

ratio for the deviations from circularity for both shadows. In the discussion we consider the possibility that this assumption does not hold.

\section{Altitude Gradient of Haze Density}

The observations and the theoretical calculations yield the top altitude of Titan's haze as a function of position angle as if the haze were opaque to a certain altitude and transparent above that altitude. These altitudes are shown in Fig. 3. We used Titan's surface radius of $2575 \mathrm{~km}$ (Davies et al. 1992).

Titan's haze does not have a sharp upper boundary, but decreases according to a scale height of about $45 \mathrm{~km}$ (Toon et al. 1992). Since the scale height is small compared to the smear of the shadow, our measurement refers to the effective altitude of the optical limb. The optical radius of Titan is defined as the radius of a sphere blocking the same amount of light on a screen behind Titan (or on Saturn). With this definition, Titan's extinction efficiency is unity.

\section{Observational Results}

Our observational results shown in Fig. 3 indicate three conclusions. First, the altitude decreases with increasing wavelength. Second, the decrease is much stronger near the South Pole than throughout the northern hemisphere. Third, within the noise level of 5-10 km, the shapes of the curves are similar.

In order to show the shape of the latitudinal variation with reduced noise, we also plot the average of data for 
all five wavelengths in Fig. 3, displaced by $100 \mathrm{~km}$. Morning and evening limbs were also averaged for this curve (solid curve at bottom). The dashed fitted curve is a synthetic profile which has a constant altitude north of $-5^{\circ}$ and south of $-50^{\circ}$ latitude and a linear transition region inbetween. These three latitude regions are called here northern, southern, and transition region. The synthetic profile was smeared according to the size of the Sun and the point spread function of the telescope, which rounds off the corners of the synthetic profile somewhat. The size of the Sun and the point spread function limit our latitudinal resolution to $15^{\circ}$. Our observations were easily capable of detecting features down to scales of $15^{\circ}$. The fact that we only find a large-scale feature indicates that aerosol properties and distribution are mostly governed by largescale processes such as one global Hadley circulation.

Fitting each curve by a profile of the same shape, we found the altitude of the optical limb northward of $-5^{\circ}$ latitude, $H_{\mathrm{N}}$, the altitude southward of $-50^{\circ}$ latitude, $H_{\mathrm{S}}$, and the statistical standard deviation between the observed and fitted curve, $\sigma$. These data are listed in Table I.

This section discussed a number of systematic effects of size 16-19 km (size of the Sun), $13 \mathrm{~km}$ (point spread function), and other effects smaller than $5 \mathrm{~km}$. Since we corrected our data for all major systematic effects, we estimate that our accuracy is about $15 \mathrm{~km}$. This value is much smaller than observed variations so that a number of conclusions can be made.

\section{DISCUSSION}

\section{Global Properties}

Our first conclusion is that Titan's optical radius decreases with increasing wavelength, which is well known. Toon et al. (1992) give an equation for Titan's optical radius as a function of wavelength which is shown in Fig. 4 as the dotted line. They only give a global model, independent of latitude. The dotted line runs roughly between our data points for the northern (filled circles) and southern region (open circles). They defined the optical radius as the radius where the vertical extinction optical depth is 0.05 . Our definition corresponds to grazing rays encountering an optical depth of 0.56 along their path if the scale height is constant with altitude. For a scale height of $45 \mathrm{~km}$, this is the altitude of an extinction optical depth of 0.028 . Thus, our altitudes should be $25 \mathrm{~km}$ higher than Toon's altitudes. This is indeed the case as well as one can expect for a comparison with a globally averaged model. Global averaging could be done in different ways. For example, along the limb, the southern region constitutes an arc almost half as long as the arc for the northern region. However, on the disk viewed from earth, the southern region has an area almost 10 times smaller than the northern region. Considering that the altitude of the optical limb at our

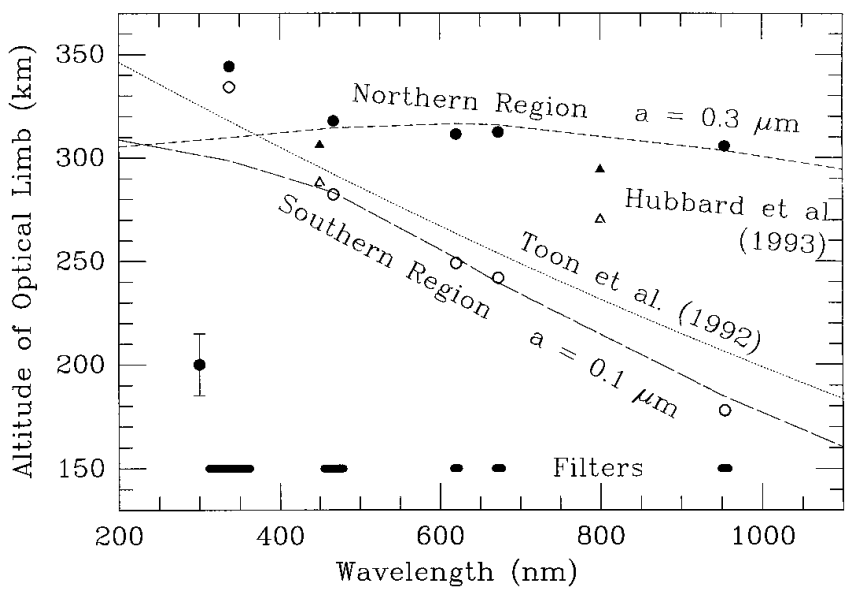

FIG. 4. Altitude of the optical limb as function of wavelength. Altitudes at northern (filled circles) and southern regions (open circles) match the expected variation for aerosol spheres of radius 0.3 and $0.1 \mathrm{~mm}$ (dashed lines), except for the shortest wavelength. This offset can be explained by a fractal shape of aerosols (see text). Data by Hubbard $e t$ al. (1993) are shown as filled triangles (north of $-20^{\circ}$ latitude) and open triangles (south of it). For comparison, the altitude function by Toon et al. (1992) is plotted as the dotted line. Horizontal bars at the bottom mark approximate filter transmission regions. The size of the $15-\mathrm{km}$ error bar for data points of this work is shown at lower left.

longest wavelength varies by three scale heights with latitude, global averaging is not recommended. Future models need to consider a latitudinal variation.

\section{Comparison with Pioneer 11}

Smith (1980) measured the radius of Titan at 440 and $640 \mathrm{~nm}$ wavelength. The corresponding altitudes are $305 \pm$ 22 and $265 \pm 25 \mathrm{~km}$. This is in agreement with our data, when averaged globally.

\section{Comparison with Voyager}

Smith et al. (1981) reported Titan's optical limb from Voyager 1 images as $240 \mathrm{~km}$ altitude, although no wavelength dependence was investigated. Using a high phaseangle image in the clear filter (480 nm effective wavelength) from Voyager 2, Rages and Pollack (1983) measured the equatorial and polar diameter. They give altitudes of the limb as 357 and $340 \mathrm{~km}$, corresponding to the level of 0.01 optical depth. Both altitudes are $42 \mathrm{~km}$ higher than our altitudes for 0.028 optical depth at the same, interpolated wavelength. This is perfectly consistent with an aerosol scale height of $45 \mathrm{~km}$. For this comparison, we averaged our data for both poles. Their centering method did not allow to find an asymmetry between both hemispheres.

The result by Rages and Pollack (1983) of higher extinction near the equator could be interpreted in two ways-as an enhanced number density there, or the same number density of larger aerosols. The former situation would 
cause all wavelengths to show the same or a similar latitudinal variation. Our observations indicate that this is not the case. Rages and Pollack (1983) used only a clear filter, so they had no information at different wavelengths (although their analysis could perhaps be repeated using Voyager images taken with different filters).

Rages and Pollack (1983) fit both a circle and an ellipse to their estimates of extinction altitude, and noted that an ellipse fitted significantly better. Since they lacked a fiducial marker from which to estimate the true location of Titan's center, they were unable to discriminate an ellipse from a circle with a flattened or notched top, which is our interpretation of our data.

\section{Aerosol Sizes}

We interpret our data by a variation of size of aerosols with latitude. The dashed curves of Fig. 4 show our calculations. We used a scale height of $45 \mathrm{~km}$ for the haze, an altitude of $265 \mathrm{~km}$ for extinction efficiency of unity, and two sizes, 0.1 and $0.3 \mu \mathrm{m}$ (area-weighted), for mean radii. We used Mie theory, a Hansen size distribution with a normalized variance of 0.2 , and optical constants by Toon et al. (1992) listed here in Table I. These constants were based on Khare et al. (1984) with a small adjustment to match Titan's albedo. The data points for the southern and northern regions mostly follow these curves. Thus, the southern region has smaller aerosols than the northern region, but the geometric optical depth is similar at all latitudes (geometric optical depth is the optical depth for extinction efficiency of unity, thus it is the macroscopic optical depth if the wave nature of light were ignored).

The latter conclusion must be seen in the context of our north-south centering method. We accurately know relative positions of Titan from its velocity vector, but not absolute positions. If we had assumed another north-south position, altitudes at all wavelengths would be shifted by the same amount up for one pole and down for the other pole. This would not change derived aerosol sizes since sizes are dependent on the variation of altitude with wavelength, but it would introduce a sinusoidal latitudinal variation of the altitude of the optical limb for extinction efficiency of unity, centered at $265 \mathrm{~km}$ altitude. Thus, our observations cannot exclude such a pure sinusoidal variation, but can exclude variations of other shapes with amplitudes in excess of $15 \mathrm{~km}$.

The best fitting sizes for both hemispheres are almost independent of the assumed imaginary refractive index and only slightly dependent on the assumed real refractive index. This result can be understood by comparing the last two columns of Table I. A factor of three in size changes the extinction efficiency by a factor of 20 . On the other hand, optical properties change extinction efficiencies only by small amounts. Clearly, our data are far more sensitive to size than to optical parameters. On the other hand, Titan's albedo is quite dependent on both size and optical parameters.

Our data points for the northern region indicate an altitude almost constant with wavelength. This is only possible for an aerosol size near $0.3 \mu \mathrm{m}$ or larger, even if the aerosol scale height were different. Slightly larger sizes would require a positive correlation of wavelength and altitude which is not observed. Sizes much larger than $0.3 \mu \mathrm{m}$ are unlikely. They are in conflict with previous observations. The best fitting size is almost independent of the assumed normalized variance of the size distribution.

The derived aerosol size for the southern region is very dependent on the assumed aerosol scale height $(45 \mathrm{~km})$. According to Toon et al. (1992), the aerosol scale height is similar, though rather slightly smaller than the gas density scale height. The gas density scale height is $50 \mathrm{~km}$ at altitudes of 200-300 km (Lellouch and Hunten 1989). Toon et al. (1992) found scale heights larger than $50 \mathrm{~km}$ only for models with upward winds, but Hourdin et al. (1995) predict downward winds for the winter (southern) region. An aerosol scale height of $50 \mathrm{~km}$ yields a best fit for radius $0.11 \mu \mathrm{m}$. Our assumed value of $45 \mathrm{~km}$ yields radius 0.10 $\mu \mathrm{m}$. A scale height less than $45 \mathrm{~km}$ yields southern aerosols smaller than radius $0.1 \mu \mathrm{m}$. The limiting case is a scale height of $37 \mathrm{~km}$ where the wavelength dependence becomes $\lambda^{-4}$ which is that of Rayleigh extinction, requiring very small aerosols. While our observations cannot exclude this case, we do not favor a latitudinal variation of aerosol sizes of more than the minimum required factor of 3 . The derived aerosol size is somewhat dependent on the assumed normalized variance $b$ of the size distribution (0.2). An assumed value of $b=0.1$ would increase the mean size to $0.12 \mu \mathrm{m}$, while $b=0.4$ gives a size of $0.08 \mu \mathrm{m}$.

Physical models of Titan's atmosphere by Toon et al. (1992) yielded global aerosol radii near $0.15 \mu \mathrm{m}$, based on data at several of Titan's seasons. A curve in Fig. 4 for this size would plot about halfway between the curves for 0.1 and $0.3 \mu \mathrm{m}$ radius. Thus, their size is in as good agreement with ours as possible for a global number.

\section{Varying Aerosol Scale Height}

Voyager 2 images analyzed by Rages and Pollack (1983) display a distinct detached haze layer at altitude 300-350 $\mathrm{km}$. Their extinction profiles show that just below this layer, aerosol extinction decreases for some $30 \mathrm{~km}$ by up to a factor of 2, while it increases moving down at other altitudes. Near this reversal of extinction gradient, the assumption of an aerosol scale height constant with altitude is clearly wrong. When calculating the extinction cross section of Titan with profiles by Rages and Pollack (1983) scaled to different extinction efficiencies corresponding to different wavelengths, a small reversal of the otherwise 
decreasing aerosol extinction with altitude is almost indistinguishable from an atmosphere with constant scale height. Their average aerosol scale height in the altitude region $200-400 \mathrm{~km}$ is about $50 \mathrm{~km}$, similar to our assumed value of $45 \mathrm{~km}$. Thus, while Titan's aerosol scale height is not constant, shadow data are not sensitive to details of the vertical extinction profile. Small variations of aerosol scale heights with altitude and/or latitude would shift our best-fitting sizes by a small amount compared to the observed north-south asymmetry in aerosol sizes.

It should be noted that our data could be explained by constant aerosol sizes. This would require a latitudinal variation of aerosol scale height of almost a factor of 10 throughout the altitude range $200-400 \mathrm{~km}$. However, Rages and Pollack (1983) find almost no variation of average scale height with latitude in this altitude region, so a constant aerosol size seems very improbable.

\section{Comparison with Stellar Occultation}

Hubbard et al. (1993) analyzed a large set of observations of the occultation of $28 \mathrm{Sgr}$ by Titan in 1989. They found a thicker haze north of $-20^{\circ}$ latitude than south of it. We inferred the altitude of the optical limb from their Fig. 6, and these data are plotted here in Fig. 4 as the triangles. Their and our data sets agree that the southern hemisphere has the lower optical limb, but the altitudes do not quite agree. Hubbard et al. (1993) concluded that the seasonal reversal took place between 1981 and 1989. Thus, there should not be a seasonal reversal between 1989 and 1995, and this is indeed the case. However, the atmosphere did change somewhat during these six years as can be seen from the change of altitudes of optical limb. It seems that the reversal took place near 1987, the solstice, and by 1989 , the north-south asymmetry was not yet built up to full amplitude as in 1995. This is consistent with the phase lag seen in the north-south asymmetry of the albedo (Lorenz et al. 1997).

Hubbard et al. (1993) also determined a mean aerosol size from the wavelength dependence of extinction. For a normalized variance of 0.2 , their aerosol radius is $0.1 \mu \mathrm{m}$. This is consistent with ours for the southern region. Their aerosol size may mostly reflect that of southernhemisphere aerosols, since their observations of the southern latitudes include wavelengths between 0.36 and 0.89 $\mu \mathrm{m}$, while the observations of latitudes well north of the equator only have a small wavelength range of $0.53-0.72$ $\mu \mathrm{m}$. Hubbard et al. (1993) assumed an aerosol scale height of $30 \mathrm{~km}$ while we assume a scale height of $45 \mathrm{~km}$. A smaller assumed scale height yields smaller aerosol sizes which is another explanation for their smaller average size compared to ours. As explained above, our observations put a lower limit on the aerosol scale height for the southern region in 1995 at $37 \mathrm{~km}$.
The aerosol scale height is the only parameter of Titan's atmosphere needed to infer relative extinction efficiencies of aerosols as a function of wavelength from our observations. Our method is a very direct method to infer aerosol extinction and aerosol size.

On the other hand, occultation data are very sensitive to the gas density and density gradient. Thus, Hubbard's haze model is somewhat simpler than ours, assuming constant properties northward and southward of $-20^{\circ}$ latitude and assuming globally constant aerosol size. Nevertheless, both data sets together provide one of the best indications of physical variations with Titan's seasons.

\section{Aerosol Shape}

Altitude data for both hemispheres fit the dashed curves of Fig. 4 only for four out of the five wavelengths. The ultraviolet data indicate a $30 \mathrm{~km}$ higher altitude than the fitted curve for both hemispheres. This difference is well above our accuracy and thus significant.

Such a discrepancy is not new. Models which fit observed geometric albedos well in visible and near-infrared continuum and methane bands tend to be too bright in the ultraviolet. More ultraviolet opacity is needed to shield reflection from bright Rayleigh scattering. Rannou et al. (1995) showed that models with aerosols as fractals instead of spheres can fit the geometric albedo spectrum since fractals show an enhanced ultraviolet extinction efficiency compared to spheres of the same volume. For example, Fig. 4 by Rannou et al. (1995) shows that fractals of fractal dimension 2 consisting of 16 spheres have identical extinction efficiency as spheres of the same volume at wavelengths above $400 \mathrm{~nm}$, but an increased ultraviolet extinction efficiency of a factor of about 1.3. This would lift the calculated optical limb at the ultraviolet wavelength by $10-15 \mathrm{~km}$, in better agreement with the observations, but not quite sufficient. For fractals of 256 monomers, the ultraviolet increase is up to a factor of 4 in extinction efficiency or an increase of optical limb of some $60 \mathrm{~km}$, which is more than observed. Fractals with a number of monomers somewhere between 16 and 256 may be able to fit our data quite well.

Such fractals are similar to those proposed by West and Smith (1991). Their fractals consist of monomers of radius $0.06 \mu \mathrm{m}$, and the fractals' mean projected area is equal to that of a sphere of radius $0.14 \mu \mathrm{m}$. This was based on polarimetry of Titan, requiring an aerosol size near 0.1 $\mathrm{mm}$ if spherical (Tomasko and Smith 1982), and on the albedo at high phase angles requiring an aerosol size $>0.2$ $\mu \mathrm{m}$ if spherical (Rages et al. 1983).

\section{Images}

In Titan's image at wavelength $954 \mathrm{~nm}$, the albedo near Titan's limb is highest near the South Pole, and is similarly 
low throughout the northern hemisphere and tropical southern latitudes. There seems to be a bright polar feature south of about $-50^{\circ}$ latitude. Thus, the latitudinal pattern in albedo seems to be similar to the pattern in altitude of optical limb and thus aerosol size. Also, at the opposite season, Voyager blue and violet images show a dark polar hood north of $+50^{\circ}$ (Sromovsky et al. 1981). This is consistent with a bright polar hood at wavelength $954 \mathrm{~nm}$, since Voyager imaged at visible and ultraviolet wavelengths where the albedo contrast is reversed from that at infrared wavelengths. This was seen by Lorenz et al. (1997), who also investigated the north-south asymmetry in albedo as a function of time. This polar feature is not the same as the transition between the bright and dark hemisphere which is seen well near the center of the disk and thus seems to be lower in the atmosphere. The feature near $50^{\circ}$ latitude is best seen near the limb and thus is probably higher up ( $\sim 200-300 \mathrm{~km}$ altitude) and of low optical depth.

Another feature shows a similar latitudinal variation as our determined aerosol sizes, the detached haze layer. In Voyager 2 images at high phase angles, the detached haze layer is clearly seen south of $15^{\circ}$ latitude; it is still present at $30^{\circ}$ and $45^{\circ}$, but at $60^{\circ}$ North it is gone completely. Because of the hemispheric reversal, our northern region is now the region of the detached haze layer, while our southern region lacks a detached haze layer. We determined an aerosol size of $0.3 \mu \mathrm{m}$ at the latitudes of the detached haze layer, and a size of $0.1 \mu \mathrm{m}$ where the detached haze layer is not present. The transition region for aerosol sizes is almost the same as the transition region for the presence of the detached haze layer. This suggests that the detached haze layer consist of aerosols of size 0.3 $\mu \mathrm{m}$, while the main haze layer below has aerosols of size $0.1 \mu \mathrm{m}$.

Tomasko and Smith (1982) suggested that a layer of larger aerosols overlies the main haze layer of smaller aerosols. The revised model by Toon et al. (1992) follows this suggestion and has a jump in the aerosol size from $0.05 \mu \mathrm{m}$ just below approximately $300 \mathrm{~km}$ altitude to 0.25 $\mu \mathrm{m}$ just above. Because their size profile has a narrow peak at $0.25 \mu \mathrm{m}$, shadow measurements would yield a size well below $0.25 \mu \mathrm{m}$. While our aerosol sizes are larger, the general agreement between model and observation is satisfactory.

The detached haze layer lies at an altitude of 300-350 $\mathrm{km}$ and has a vertical optical depth of $\sim 0.04$ according to Toon et al. (1992). Shadow measurements of the northern region do not probe much below this level. Thus, our observations of the northern region are mostly sensitive to the detached haze layer. The main haze layer below may have aerosols as small as those seen in the southern region.

Smith et al. (1996) inferred Titan's surface albedo features from images near wavelengths 940 and $1020 \mathrm{~nm}$. The determination of the contrast of surface features requires the knowledge of the optical depth of the overlying haze. Our observations indicate that the optical depth at these wavelengths varies by a factor of 20 with latitude at altitudes near $300 \mathrm{~km}$ (extinction efficiencies are listed in Table I). However, Lemmon (1994) noted that the southern hemisphere has the larger haze optical depth derived from methane band images probing to much lower altitudes, near $100 \mathrm{~km}$. Thus, our measurements of aerosol sizes and optical depths at altitudes near $300 \mathrm{~km}$ are not representative of the main haze layer below.

\section{Titan's Circulation}

Hourdin et al. (1995) presented a global circulation model of Titan's atmosphere. Near equinoxes, when our observation took place, their model predicts upwelling motion near the equator and downwelling motion near both poles (cf. their Fig. 6). Their model does not explain the observed north-south asymmetry. Their model of northern winter solstice (2002) shows upwelling in the south and downwelling in the north: we assume that the situation during the recent northern summer is the reverse, with gas rising in the north hemisphere and descending in the south. Such a circulation increases the number of aerosols in the main haze layer in the south and levitates the detached haze in the north. This is what we observe in 1995. Thus, Titan's distribution of aerosols seems to lag that inferred from winds predicted by Hourdin et al. (1995). The lag in the behavior of the detached haze is consistent with the north-south asymmetry in albedo examined by Sromovsky et al. (1981) and Lorenz et al. (1997).

Another two-dimensional haze model was investigated by Hutzell et al. (1996). For the present season, they predict upwelling motion in the southern hemisphere and larger aerosol sizes there than in the northern hemisphere, opposite to our findings. They assumed a boundary layer at 300 $\mathrm{km}$ altitude and restrict their calculations to altitudes below that layer. Their model may correctly represent winds and aerosol sizes at lower altitudes, well below $300 \mathrm{~km}$, but it clearly is not appropriate for altitudes near $300 \mathrm{~km}$, which our observations probe. Their model is also only modestly successful at reproducing the observed albedo contrast between hemispheres.

With more progress in modeling, we may start to understand dynamics in Titan's atmosphere. On the other hand, another observation of Titan's shadow will have to wait until 2008. Near 2008, the Cassini spacecraft will give us accurate measurements of Titan's atmosphere at the season opposite from that in 1995.

\section{Limb Measurements in Images}

We measured the location of the limb in our images of Titan, trying to account for different albedos at different latitudes, and compared it with the theoretical motion of 
Titan's center of gravity. We find an apparent offset of Titan's center of $60 \mathrm{~km}$ between our shortest and longest wavelengths. This corresponds to an increase of the northsouth difference in radius of $120 \mathrm{~km}$ over our wavelength range, the same value as observed for the shadows. Also, the offset in the images at wavelengths 620 and $673 \mathrm{~nm}$ is about halfway between our extreme wavelengths, which is also similar to the shadows.

However, it is difficult to estimate the systematic error of limb measurements in the images for three reasons. First, the albedo of aerosols changes with latitude. Second, the determination is critically dependent on the limb darkening and the point spread function of the telescope which are not perfectly known. This is especially problematic for the southern region which is only two pixels wide in these images. Third, the solar illumination from the northeast makes it difficult to directly compare different latitudes. Nevertheless, the agreement of measurement between images and shadows indicates that systematic effects are probably small, and that some of our results such as the data on the north-south radius difference could have been obtained by measuring Titan's images almost as well as by measuring Titan's shadow.

Our images are the first images where a north-south asymmetry of the optical limb has been detected due to the reference position of Saturn's sharp ring. Voyager and previous Hubble Space Telescope images did not have such a reference. Thus, our main observational result, the north-south asymmetry of optical limb as a function of wavelength, was not detected before. The only previous nonimaging measurement of the north-south asymmetry in altitude came from a stellar occultation in 1989 (Hubbard et al. 1993).

\section{SUMMARY}

We measured the shape and size of Titan's shadow at five wavelengths. We inferred Titan's optical radius as a function of wavelength and latitude. We found three distinct latitude regions, a northern region northward of $-5^{\circ}$ latitude with a high optical limb, a southern region southward of $-50^{\circ}$ with a low optical limb, and a linear transition region inbetween. There are no other significant features present on scales down to $15 \mathrm{~km}$.

The variation of altitude with latitude increases with wavelength, from close to zero at $337 \mathrm{~nm}$ to $130 \mathrm{~km}$ at 954 $\mathrm{nm}$. We interpret this variation by a variation of aerosol sizes, from $0.1 \mu \mathrm{m}$ in the southern region to $0.3 \mu \mathrm{m}$ in the northern region. Our observations are consistent with a constant geometric optical depth for all latitudes, measured at the same altitude. The altitude of the optical limb is 265 $\mathrm{km}$ for extinction efficiency of unity. Considering the size variation of a factor of 3 , the southern region has a $\sim 9$ times higher aerosol number density but a $\sim 3$ times smaller aerosol volume density than the northern region at the same altitude.

Our observations mostly probe aerosols at altitudes near $300 \mathrm{~km}$, where Voyager recorded a detached haze layer. This layer was present throughout the southern hemisphere, gradually decreasing north of the equator and vanishing in mid-northern latitudes where the main haze layer below seemed to extend to higher altitudes. Taking into account seasonal reversal between the Voyager encounters and our observations, this latitudinal distribution matches our distribution of aerosol sizes with latitude almost perfectly. This suggests that we measured aerosol sizes of two distinct haze layers. Thus, the main and detached haze layers have aerosol sizes of 0.1 and $0.3 \mu \mathrm{m}$, respectively.

Observations at four out of our five wavelengths are consistent with spherical aerosols. However, the ultraviolet data require an extinction efficiency significantly larger than that for spherical particles. This is indicative of a fractal shapes of aerosols. Only certain fractals are consistent with our data (between 16 and 256 monomers/ particle).

An equatorial bulge of $17 \pm 11 \mathrm{~km}$ was seen in Voyager images (Rages and Pollack 1983), very consistent with our data interpolated to the same wavelength $(480 \mathrm{~nm})$. Voyager's spectral coverage was not sufficient to distinguish between a latitudinal variation of aerosol amount and a variation of aerosol size, nor did the images allow for the detection of the north-south asymmetry of extinction.

A comparison with data on aerosol size and optical depth from a stellar occultation in 1989 (Hubbard et al. 1993) shows that the north-south asymmetry increased since 1989, indicating that the hemispherical reversal may have taken place near or before the solstice in 1987. This is consistent with the phase of the north-south asymmetry in albedo (Lorenz et al. 1997).

Our detected latitudinal variation of the altitude of the optical limb of $130 \mathrm{~km}$ is a large effect. It corresponds to almost 3 scale heights, or a factor of 15 in pressure. The detection was possible due to an advantage of our images over Voyager and previous Hubble Space Telescope images of Titan. We imaged Titan's shadow together with sharp, nearly edge-on rings which provided the positional reference between different wavelengths. The missing reference location in previous images prevented the detection of the large asymmetry in altitude of the optical limb.

Most previous constraints on sizes of Titan's aerosols derive from observed light reflected from Titan. These observations are sensitive to the aerosol optical depth, albedo, and phase function, all of which are wavelength dependent. Determination of optical depth by reflected light requires an estimation of albedo and phase function. The other previous method of determining aerosol sizes, using a stellar occultation by Titan, is also sensitive to the gas density and density gradient. Our observations using 
Titan's shadow are the most direct measurements of aerosol optical depths, completely independent of albedos, phase functions, and gas density.

\section{ACKNOWLEDGMENTS}

We thank Martin Tomasko for his involvement in the observations. He, Mark Lemmon, Robert West, and Chris McKay provided helpful suggestions on the manuscript. Support for this work was provided through Grant AST-9417368 from the National Science Foundation and through Grant GO060300194A from the Space Telescope Science Institute, which is operated by the Association of Universities for Research in Astronomy, Inc., under NASA Contract NAS5-26555.

\section{REFERENCES}

Allen, C. W. 1973. Astrophysical Quantities, 3rd. ed. Athlone Press, London.

Biretta, J. 1995. WFPC2 flat field calibration. In Calibrating Hubble Space Telescope: Post Servicing Mission (A. Koratkar and C. Leitherer, Eds.). Space Telescope Science Institute, Baltimore, MD.

Burrows, C. J., S. M. Baggett, J. Biretta, S. Casertano, M. Clampin, R. E. Griffiths, AND J. KRIST 1995. Hubble Space Telescope Wide Field and Planetary Camera 2 Instrument Handbook. Space Telescope Science Institute, Baltimore, MD.

Davies, M. E., V. K. Abalakin, A. Brahic, M. Bursa, B. H. Chovitz, J. H. Lieske, P. K. Seidelmann, A. T. Sinclair, And Y. S. Tuuflin 1992. Report of the IAU/IAG/COSPAR working group on cartographic coordinates and rotational elements of the planets and satellites: 1991. Celest. Mech. Dynam. Astron. 53, 377-397.

Flasar, F. M., R. E. Samuelson, and B. J. Conrath 1981. Titan's atmosphere: Temperature and dynamics. Nature 292, 693-698.

Hourdin, F., O. TAlagrand, AND R. SADOURny 1995. Numerical simulation of the general circulation of the atmosphere of Titan. Icarus 117, 358-374.

Hubbard, W. B., G. J. Consolmagno, R. P. Boyle, and R. Hill 1996. The shadow of Titan on Mimas and Saturn's rings. Proc. Lunar Planet. Sci. Conf. 27, 569-570. [Abstract]

Hubbard, W. B., B. Sicardy, R. Miles, A. J. Hollis, R. W. Forrest, I. K. M. Nicolson, G. Appleby, W. Beisker, C. Bittner, H.-J. Bode, M. Bruns, H. Denzau, M. Nezel, E. Riedel, H. Struckmann, J. E. Arlot, F. Roques, F. Sèvre, W. Thuillot, M. Hoffmann, E. H. Geyer, C. Buil, F. Colas, J. Lecacheux, A. Klotz, E. Thouvenot, J. L. Vidal, E. Carreira, F. Rossi, C. Blanco, S. Cristaldi, Y. Nevo, H. J. Reitsema, N. Brosch, K. Cernis, K. Zdanavicius, L. H. Wasserman, D. M. Hunten, D. Gautier, E. Lellouch, R. V. Yelle, B. Rizk, F. M. Flasar, C. C. Porco, D. Toublanc, and G. Corugedo 1993. The occultation of $28 \mathrm{Sgr}$ by Titan. Astron. Astrophys. 269, $541-563$.

Hutzell, W. T., C. P. McKay, O. B. Toon, And F. Hourdin 1996. Simulations of Titan's brightness by a two-dimensional haze model. Icarus 119, 112-129.

KARKoschKA, E., 1994. Spectrophotometry of the jovian planets and
Titan at 300- to 1000-nm wavelength: The methane spectrum. Icarus 111, 174-192.

KarkoschKa, E., And M. Tomasko 1993. Saturn's upper atmospheric hazes observed by the Hubble Space Telescope. Icarus 106, 428-441.

Khare, B. N., C. Sagan, E. T. Arakawa, F. Suits, T. A. Callcott, and M. W. Williams 1984. Optical constants of organic tholins produced in a simulated titanian atmosphere: From soft X-ray to microwave frequencies. Icarus 60, 127-137.

Krist, J. 1994. The Tiny Tim User's Manual, Version 4.0. Space Telescope Science Institute, Baltimore, MD.

Lellouch, E., AND D. M. Hunten 1989. Titan Atmosphere Engineering Model. Space Science Department of ESA, ESLAB 87/109.

Lemmon, M. T. 1994. Properties of Titan's haze and surface. Ph.D. Thesis, Univ. of Arizona.

Lorenz, R. D., P. H. Smith, M. T. Lemmon, E. Karkoschka, G. W. LockWOOD AND J. D. CALDWELL 1997. Titan's north-south asymmetry from Hubble Space Telescope and Voyager imaging: Comparison with models and groundbased photometry. Icarus, in press.

NeCKEL, H., AND D. LABs 1984. The solar radiation between 3300 and 12500 A. Solar Phys. 90, 205-258.

Rages, K., And J. B. Pollack 1983. Vertical distribution of scattering hazes in Titan's upper atmosphere. Icarus 55, 60-62.

Rages, K., J. B. Pollack, AND P. H. Smith 1983. Size estimates of Titan's aerosols based on Voyager high-phase-angle images. J. Geophys. Res. 88, 8721-8728.

Rannou, P., M. Cabane, E. Chassefiere, R. Botet, C. P. McKay, and R. Courtin 1995. Titan's geometric albedo: Role of the fractal structure of the aerosols. Icarus 118, 355-372.

Smith, B. A., L. Soderblom, R. Beebe, J. Boyce, G. Briggs, A. Bunker, S. A. Collins, C. J. Hansen, T. V. Johnson, J. L. Mitchell, R. J. Terrile, M. Carr, A. F. Cook II, J. Cuzzi, J. B. Pollack, G. E. Danielson, A. Ingersoll, N. E. Davies, G. E. Hunt, H. Masursky, E. Shoemaker, D. Morrison, T. Owen, C. Sagen, J. Veverka, R. Strom, And V. E. SuOmi 1981. Encounter with Saturn: Voyager 1 imaging science results. Science 212, 163-190.

Sмiтh, P. H. 1980. The radius of Titan from Pioneer Saturn data. $J$. Geophys. Res. 85, 5943-5947.

Smith, P. H ., M. T. Lemmon, R. D. Lorenz, L. A. Sromovsky, J. J. CAldwell, And M. D. Allison 1996. Titan's surface, revealed by HST imaging. Icarus 119, 336-349.

Sromovsky, L. A., V. E. Suomi, J. B. Pollack, R. J. Krauss, S. S. Limaye, T. Owen, H. E. Revercomb, And C. SAgAn 1981. Implications of Titan's north-south brightness asymmetry. Nature 292, 698-702.

Tomasko, M. G., AND P. H. SMITH 1982. Photometry and polarimetry of Titan: Pioneer 11 observations and their implications for aerosol properties. Icarus 51, 65-95.

Toon, O. B., C. P. McKay, C. A. Griffith, and R. P. Turco 1992. A physical model of Titan's aerosols. Icarus 95, 24-53.

Trauger, J. T., A. H. Vaughan, R. W. Evans, And D. C. Moody 1995. Geometry of the WFPC2 focal plane. In Calibrating Hubble Space Telescope: Post Service Mission (A. Koratkar and C. Leitherer, Eds.). Space Telescope Science Institute, Baltimore, MD.

West, R. A., AND P. H. SMith 1991. Evidence for aggregate particles in the atmospheres of Titan and Jupiter. Icarus 90, 330-333. 